C2020. This manuscript version is made available under the CC-BY-NC-ND 4.0 license http://creativecommons.org/licenses/by-nc-nd/4.0/

\title{
Analysis of omeprazole and esomeprazole obtained from traditional pharmacies and unlicensed internet websites using Raman spectroscopy, ${ }^{1} \mathrm{H}-\mathrm{NMR}$ and chemometric analysis
}

Gaetano F. Bellia ${ }^{a}$, Guzman Gil-Ramirez ${ }^{b}$, Mark G. Baron ${ }^{b}$, Ruth Croxton ${ }^{\text {b }}$, Jose Gonzalez-Rodriguez ${ }^{\mathrm{b}, *}$

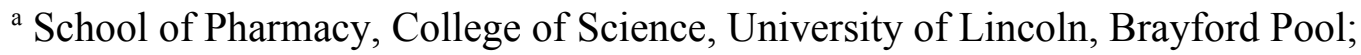

b School of Chemistry, College of Science, University of Lincoln, Brayford Pool, Lincoln, LN6 7TS, UK. Telephone: +441522886878.

* E-mail: jgonzalezrodriguez@lincoln.ac.uk

\begin{abstract}
The relevant therapeutic outcomes obtained with the use of proton pump inhibitors for the treatment of gastric conditions have an important reflection on the prescribing habits of clinicians of primary and secondary care organizations. With the attempt to reduce their financial impact on the health systems, generic medications, obtained both from tradition distribution chain and from parallel import, have represented the main type of proton pump inhibitors used in clinics recently. The effect of parallel import and the use of the internet as an international distribution network significantly facilitates the entry of falsified medicines in the official channels of distribution. Since the interchangeability of different omeprazole preparations has been previously questioned, in this study we used Raman and ${ }^{1} \mathrm{H}-\mathrm{NMR}$ to characterise different omeprazole and esomeprazole formulations produced by different manufacturers and obtained from both traditional pharmacies and from unlicensed internet market in order to establish if any differences could be found to substantiate any different clinical performances. A chemometric study of Raman spectra allowed partial separation indicating that there are not sufficient chemical differences for PCA groups to be established using this analytical approach and for these specific medications. On the contrary, NMR
\end{abstract}


investigations allowed the identification of impurities in internet samples, not detected by the Raman analyses, making this technique a valid candidate analytical tool to adopt for the investigation of falsified medicines.

\section{Keywords}

Omeprazole, generic, falsified medicines, unlicensed internet market, Raman, NMR.

\section{Introduction}

Proton pump inhibitors (PPIs) are a family of medications that block or diminish the production of gastric acid acting on the proton pump present in gastric parietal cells [1]. Omeprazole (Fig.1) and esomeprazole belong to such a family, being omeprazole a racemic mixture and esomeprazole the related Levo-enantiomer [2]. In the intracellular canaliculi of the parietal cell, particularly acidic, omeprazole is concentrated and converted to the active form, being a weak base, and interacts with $\mathrm{H}^{+}-\mathrm{K}^{+}$-ATPase, the proton pump, reversibly reducing or blocking its activity [3]. This influences the final step of the gastric acid secretion process and acts irrespectively both in the basal acid secretion and in the one instigated by stimuli. PPIs are quickly active and with a unique daily dose can maintain control of the inhibition of the gastric acid secretion [2].<smiles>[Z]c1nc(CS(=O)c2nc3cc(OC)ccc3[nH]2)c(C)c(OC)c1[2H]</smiles>

(A)<smiles>[2H]c1c([3H])nc(S(=O)c2nc3ccc(OC)cc3[nH]2)c(C)c1OC</smiles>

(B)

Fig. 1 (a) Omeprazole and (b) esomeprazole, chemical structure, with the $S$ being the chiral centre 
Because the reflux of the acidic gastric content into the oesophagus plays a major role in the pathogenesis of symptoms of GERD (gastro-oesophagal reflux disease) and lesions of erosive oesophagitis, acid suppression with a PPI is currently a mainstay of anti-reflux therapy $[3,4]$. There is a strong correlation between the degree of acid suppression provided by a given drug and its efficacy. The superiority of PPIs over other drugs (antacids, prokinetics and H2-receptor antagonists) has now been established beyond doubt, both for short- and long-term treatment [5]. Nevertheless, patients with erosive esophagitis are more reactive to PPIs than those with non-erosive reflux. Sometimes the action of PPIs in patients with atypical gastric condition is reduced to a reduction of the symptomatology of heartburn. Different studies conducted on the safety of use of PPIs both in short and long periods have provided reassuring conclusions on their safety [6-9]. PPIs have shown improved healing yield in case of severe erosive esophagitis, with quicker relief of the associated symptomatology [9]. The successful results obtained with the use of PPIs for the treatment of erosive esophagitis is having an important reflection on the prescribing habits of clinicians of primary and secondary care organizations in the United Kingdom [10]. The vast therapeutic success of PPIs has incredibly increased their use both in primary and in secondary care. With the attempt to reduce their financial impact on the health system, generic medications, obtained both from tradition distribution chain and from online import, have represented the main type of PPIs used in clinics recently $[11,12]$.

The effect of parallel import and the use of the internet as an international distribution network significantly facilitates the entry of forged medications in the official channels of distribution [13]. The Food and Drug Administration (FDA) released a study related to the effect of the internet market on the diffusion of counterfeit medications. $[14,15]$. Based on a report from WHO [16, 17], half of the medications traded on the internet sites are fakes. Because of the anonymous nature of the online market, the risks that criminals run operating in this sector is considerably high, being the investigations performed by the law enforcement authorities significantly more difficult and consequently less effective [18].

The Falsified Medicines Directive (FMD), delegated regulation 2011/62/EU [13], is a European Union legislation which aims to protect patients by preventing falsified medicines from entering the supply chain improving the security of the production and 
delivery of medications across Europe. Regulatory authorities continue to locate falsified medicines in the official supply chain, although unlicensed internet sites have now become the main route for trading falsified medicines to the public. Under the FMD, all new containers of medicines commercialised in Europe from February 2019, will present two safety features: an anti-tamper device (ATD) and a unique identifier (UI) code, in the form of a 2D data matrix barcode. Nearly all prescription-only medicines (POM) with a marketing authorisation are in the scope of FMD, with only a few exclusions. All non-prescription medicines are out-of-scope, with the exception of two omeprazole products, which were subject to falsification in the past [13]

Gastroenterology is one of the clinical areas where there are concerns among health-care professionals and patients that not all generic medications can be equally clinically effective [1]. Several clinical trials have been performed comparing different generics, in order to evaluate any therapeutic differences. In [8, 9] Shimatania et al compared, in prospective, randomized, open-label, crossover studies, the acid-suppressive effect of generic omeprazole [8] and lansoprazole [9] with that of the original brand. In both studies, the intragastric $\mathrm{pH}$ was measured at the regular interval of time. Values of stomach acidity showed figures above $\mathrm{pH} 4$ for $24 \mathrm{~h}$ cycles. These were significantly higher with the administration of any PPI formulations compared with a placebo, indicating significant differences in acid-suppression effects amongst the respective groups of PPIs. This led to the conclusion that acid-suppressive effects of some brands of generic omeprazole and lansoprazole are not the same as the original ones.

Besides these clinical studies, no information has been found in the literature concerning the chemical characterization of different PPIs, obtained both from authorised pharmacies and from non-authorised websites, aimed to investigate if different clinical features are associated with specific chemical characteristics. In fact, generic drugs are actually chemically equivalent to their brand-name counterparts or among them just in terms of active ingredients, but they may differ in peripheral features, such as inert binders and fillers, shape, colour and the specific manufacturing process, which could lead to diverse clinical responses [19-21]. This scenario highlights 
the need for the development of an analytical approach capable of discriminating amongst the different proton pump inhibitors.

Since the interchangeability of omeprazole and esomeprazole preparations produced by different manufacturers has been previously questioned, in this study, Raman and ${ }^{1} \mathrm{H}-\mathrm{NMR}$ spectroscopies have been used to characterize different omeprazole and esomeprazole formulation produced by different manufacturers and obtained from both traditional pharmacies and from the internet market in order to establish if any differences could be found to substantiate any different clinical performances.

\section{Materials and Methods}

\subsection{Materials}

Omeprazole, (5-methyl-2-\{[(4-methoxy-3,5-dimethyl-2-pyridinyl) methyl $]$ sulfonyl $\}-1 \mathrm{H}$-benzimidazole) and esomeprazole (6-methoxy-2-[(S)-(4-methoxy-3,5-dimethylpyridin-2-yl)methylsulfinyl]-1H-benzimida zole) from seven $20 \mathrm{mg}$ anonymised oral formulations (defined as A to $\mathrm{G}$ ) have been used: five of these were omeprazole (A, B obtained from traditional pharmacies, and C, D, E obtained from unlicensed internet websites) and two were esomeprazole ( F, G obtained from traditional pharmacies).

Sixteen tablets of each PPIs were analysed internally and externally by Raman and ${ }^{1} \mathrm{H}-\mathrm{NMR}$ and the results subjected to chemometric evaluations.

\subsection{Methods}

\subsubsection{Raman analysis}

Raman analysis was performed on a FORAM 685-2 instrument with a laser operating at $532 \mathrm{~nm}$. The Raman instrument was also equipped with an integral video microscope with magnification 50x. Analyses were performed between the wavenumbers 2000 and $800 \mathrm{~cm}^{-1}$. SERS-Raman enhances the sensitivity of standard Raman scattering by depositing a metal colloid on the surface of the sample being analysed. For this part of the investigation, silver colloids were prepared as follows: silver nitrate was reduced using sodium citrate in water, and concentrated by centrifuging at $5000 \mathrm{rpm}$. The eight 
tablets for SERS analysis were covered with $2 \mu \mathrm{L}$ of the prepared colloid solution and 2 $\mu \mathrm{L}$ of $\mathrm{NaCl} 1 \mathrm{M}$ as an aggregating agent. To test the external side, the tablets were coated with the silver colloid preparation and aggregating agent and exposed to the laser. This was again repeated five times for each tablet, with the laser directed at a variety of external sites, to achieve the 40 representative analyses for each PPIs. To test the internal side, the tablets were broken in half prior to the addition of the silver colloid and aggregating agent and again exposed to the laser five times for each tablet.

In order to find the optimal setting for the Raman analysis, samples were submitted to the laser exposure for different acquisition times. Spectra of the same samples were recorded at different interval of exposure time, ranging from 1 second to 5 minutes. Optimised spectra could be obtained from 60 seconds onwards of acquisition time, which was, consequently, chosen as the acquisition time for all the following analyses.

\subsubsection{Nuclear Magnetic Resonance}

${ }^{1} \mathrm{H}$ NMR spectra were obtained from a Bruker Avance 500 at $500.1 \mathrm{MHz}$ using TMS as the internal standard and MeOD as solvent. Chemical shifts for proton resonances were given in ppm $(\delta)$. Signal multiplicity was characterized by s (singlet), d (doublet) and dd (double doublet). NMR spectra were recorded using 128k complex points and a recovery time of 4 seconds 16 transients were generally sufficient to achieve good signal-to-noise. In a typical sample preparation, pills were mechanically ground to obtain a fine powder. The powder was suspended in MeOD $(1 \mathrm{~mL})$ and then filtered through a syringe filter $(45 \mu \mathrm{m})$ to obtain a solution containing the active principle. Reference samples of each of the most common excipients (lactose, cellulose, starch) were prepared in MeOD and used to identify characteristic signals in samples

\section{Results}

3.1. Raman spectroscopy of omeprazole and esomeprazole formulations

A typical SERS spectrum related to the analysis of the surfaces of the omeprazole and esomeprazole tablets investigated is shown in Fig. 2. Tablets from all the seven different formulations were analysed and analysis of the surfaces showed similar features for all samples. 


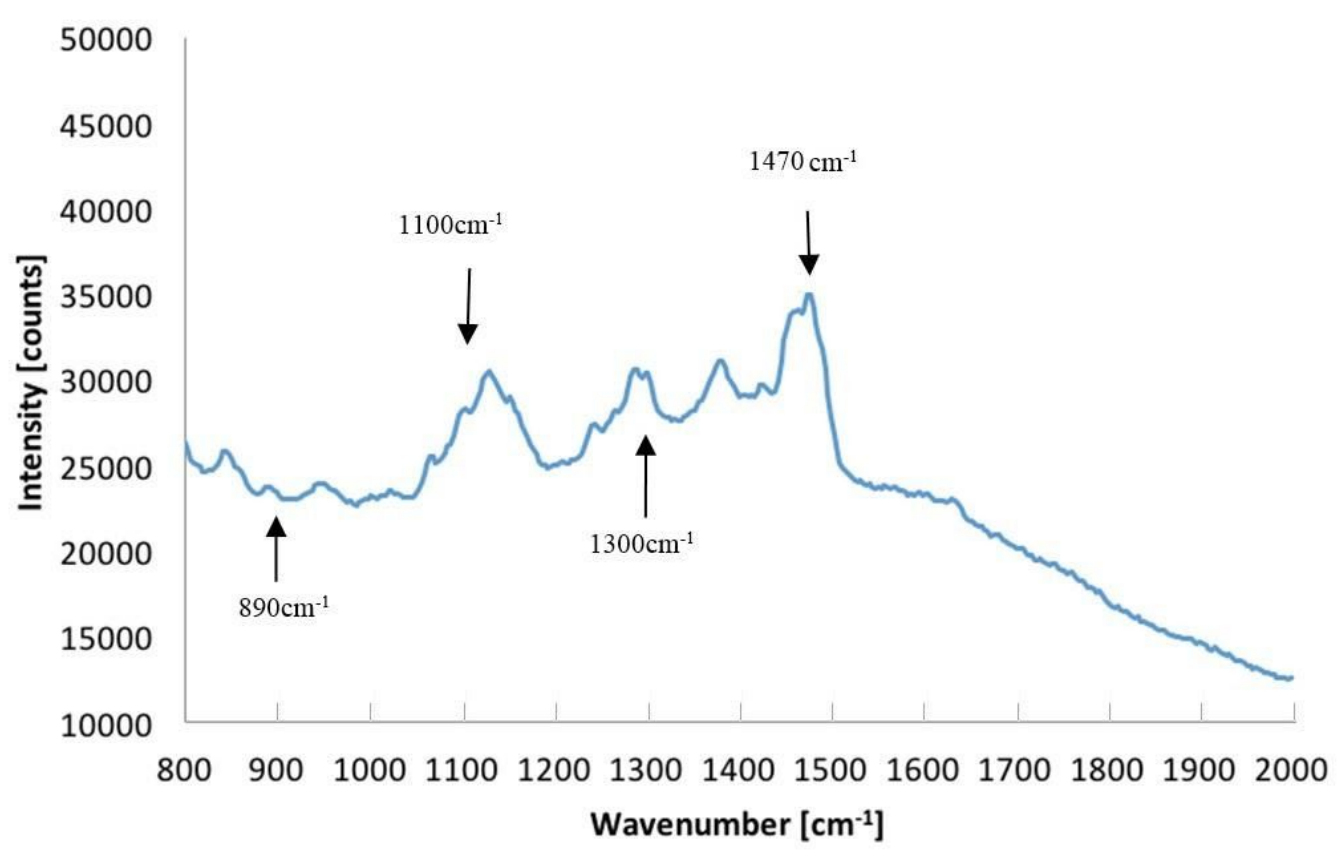

Fig. 2. Typical SERS analysis of omeprazole for sample A, external tablet surface with some of the typical bands found.

At $890 \mathrm{~cm}^{-1}$ a small but sufficiently defined peak, possibly related to the stretching of the ether groups present in the two aromatic rings of the omeprazole molecule could be observed. In the region around $950 \mathrm{~cm}^{-1}$ smooth peaks, possibly assigned to the stretching of the $\mathrm{C}-\mathrm{C}$, could be also observed. In the region around $1100 \mathrm{~cm}^{-1}$ a signal assigned to the stretching of the ether groups can also be observed. In the region around $1260 \mathrm{~cm}^{-1}$ a peak which may be referred to the stretching of the thiocarbonyl group can be observed. In the region of $1300 \mathrm{~cm}^{-1}$ a peak, possibly assigned to the symmetric stretching of the chain vibrations of the two aromatic rings, can be identified. At 1380 $\mathrm{cm}^{-1}$ a strong peak possibly due to the symmetric bending of methyl groups can also be identified. The asymmetric bending of the same groups laid in the region between 1440 and $1500 \mathrm{~cm}^{-1}$ can explain the signals recorded in the region $1460-1475 \mathrm{~cm}^{-1}$. At 1500 $\mathrm{cm}^{-1}$ a peak probably to be referred to the asymmetric bending of the methyl group. At $1632 \mathrm{~cm}^{-1}$ peak possibly related to the stretching of the aromatic rings [22, 23].

To perform the analyses of the internal part of the tablets, the seven different formulations were manually broken in half and then submitted to the preparation for 
SERS analysis as indicated previously. All formulations present signals (Fig. 3) which are slightly different from those obtained on the surface of the tablet.

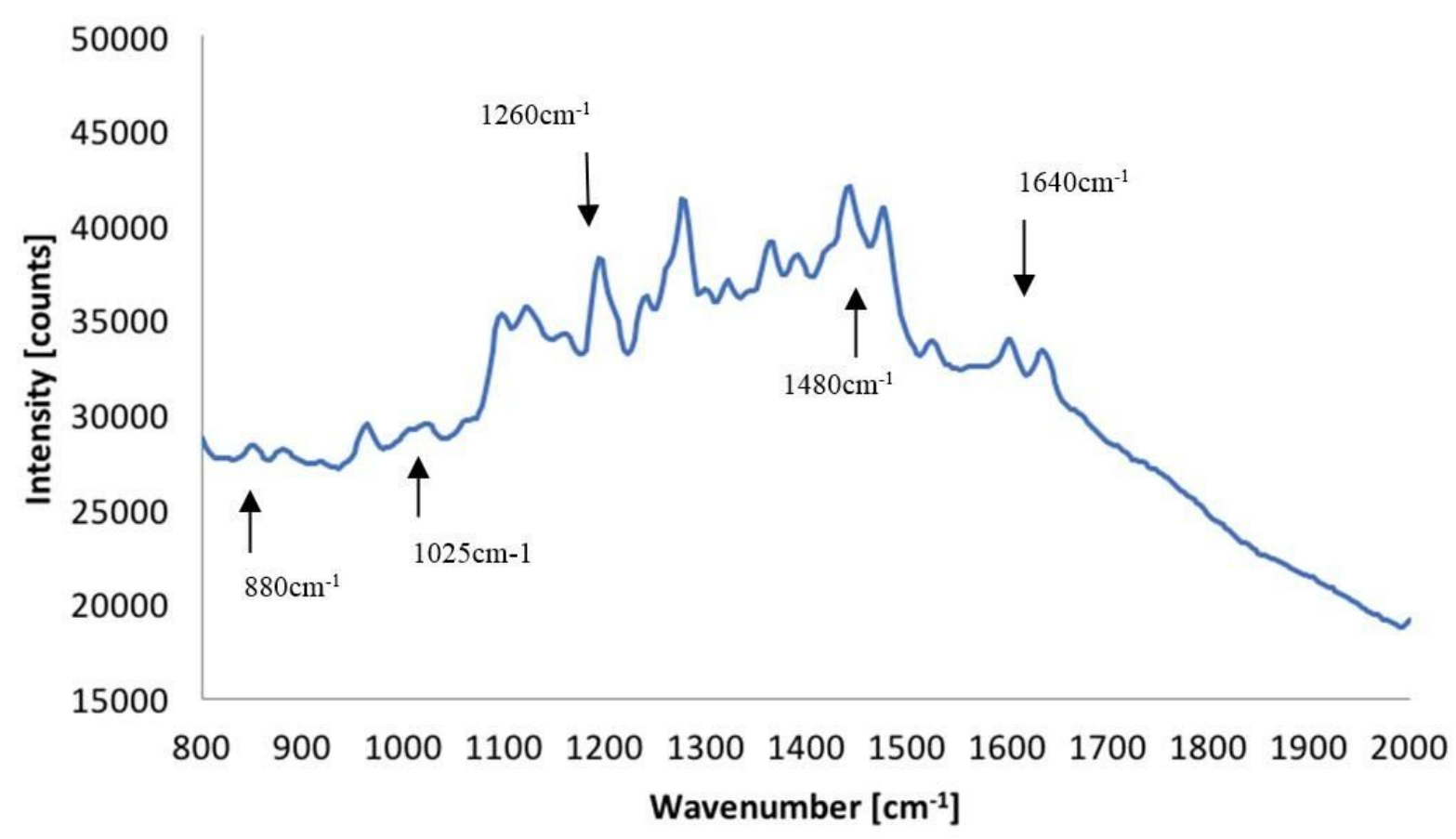

Fig. 3. Typical SERS analysis of omeprazole, sample B, internal tablet content with some of the typical bands found.

The differences found between surface and inner analyses can be attributed to a lower concentration of the drug on the surface mixed with coating agents.

The conventional Raman spectra of both the tablet surfaces and inner tablet body were also performed. These spectra show common features with those SERS previously analysed but lower intensity for main peaks (shown in figure S1 in supplementary material).

To aid in the interpretation of the vast amount of Raman shifts generated, for both SERS and conventional Raman, a Principal Component Analysis (PCA) was used (Fig. 4). The PCA scatterplots of the SERS data obtained by analysis of the external side of the omeprazole and esomeprazole tablets shows partial identifiable distribution profiles for some of the classes of samples investigated. Principal component (PC) 1 accounted 
for $93.22 \%$ of the variation, with PC2 and PC3 being responsible for 5.31\% and $0.65 \%$ respectively. PC1 and PC2 were therefore responsible for an accumulative $98.53 \%$ of the variation. The PPI formulations were separated due to their individual association with the two main components. Tablets B, F and G can be seen to partially separate over the value of PC2. G is dispersed sparsely at lower PC2 value; F is located at a central PC2 value whilst B clusters at a higher value of PC2. The cloud of data incorporating the overlap of other classes of samples is located in positive areas for both components.

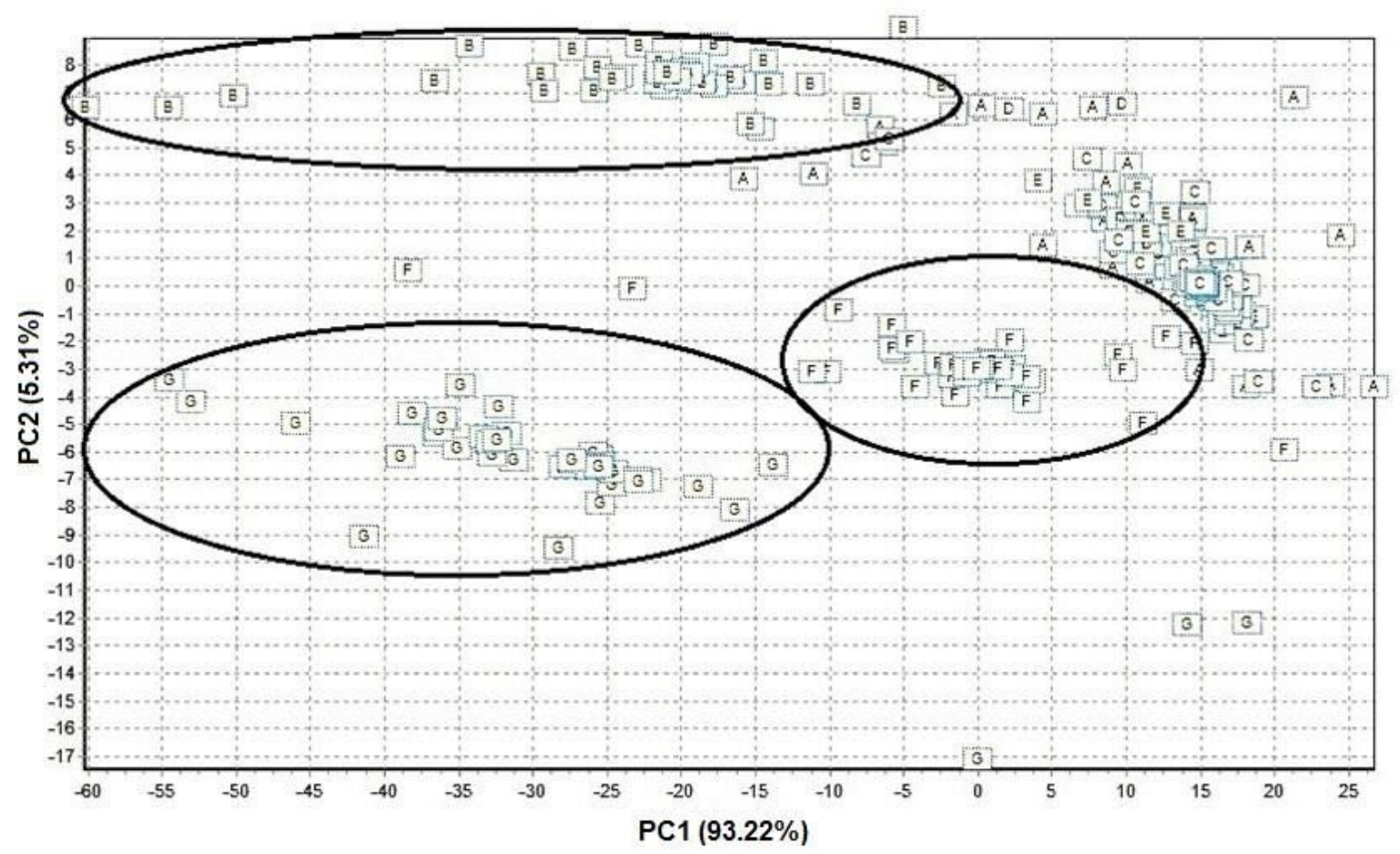

Fig. 4. Two-dimensional score plot from the Principal Component Analysis of the SERS samples for omeprazole and esomeprazole on external tablet. Omeprazole (A, B obtained from traditional pharmacies); C, D, E obtained from unlicensed internet websites); F, G (obtained from traditional pharmacies).

Predictive accuracy of the PCA model obtained was validated via cross-validation and leave-one-out approach. The total error rate of the predictive model evaluated via cross-validation accounted for $78 \%$. Furthermore, the predictive accuracy of the model was also tested via leave-one-out validation with a cumulative error rate of $80 \%$. 
PCA of the SERS data obtained by analysis of the internal side of the PPI tablets shows no clear distribution profiles for the classes of samples studied (see figure S2 in supplementary material). Principal component (PC) 1 accounted for $95.83 \%$ of variation, with $\mathrm{PC} 2$ and $\mathrm{PC} 3$ being responsible for $3.76 \%$ and $0.45 \%$ respectively. $\mathrm{PC} 1$ and PC2 were therefore responsible for an accumulative $99.59 \%$ of the variation. The total error rate of the predictive model evaluated via cross-validation accounted for $70 \%$. Leave-one-out validation on this set of data reported a cumulative error rate accounted for $75 \%$, with results comparable with the ones obtained from the cross-validation.

\section{2. ${ }^{1} \mathrm{H}-\mathrm{NMR}$ of omeprazole formulations}

In light of the similarity on some of the Raman spectra obtained and to further gather additional information on the chemical structures of the samples, the use of proton NMR was considered to elucidate more chemical diversities.

In its molecular structure, omeprazole presents two sources of structural differentiation when performing proton NMR. Firstly, omeprazole is a chiral molecule since it has a diastereogenic centre located on the thiocarbonyl sulfur atom. The second source of diversity is that it presents tautomerisms [24]

Proton nuclear magnetic resonance of a pure omeprazole standard is reported in Fig. 5. While Raman spectroscopy gives information on the functional groups present in the compound, NMR spectroscopy can determine structural features of the compounds. The omeprazole molecule presents two different aromatic rings, benzimidazole and pyridinylic moieties. 


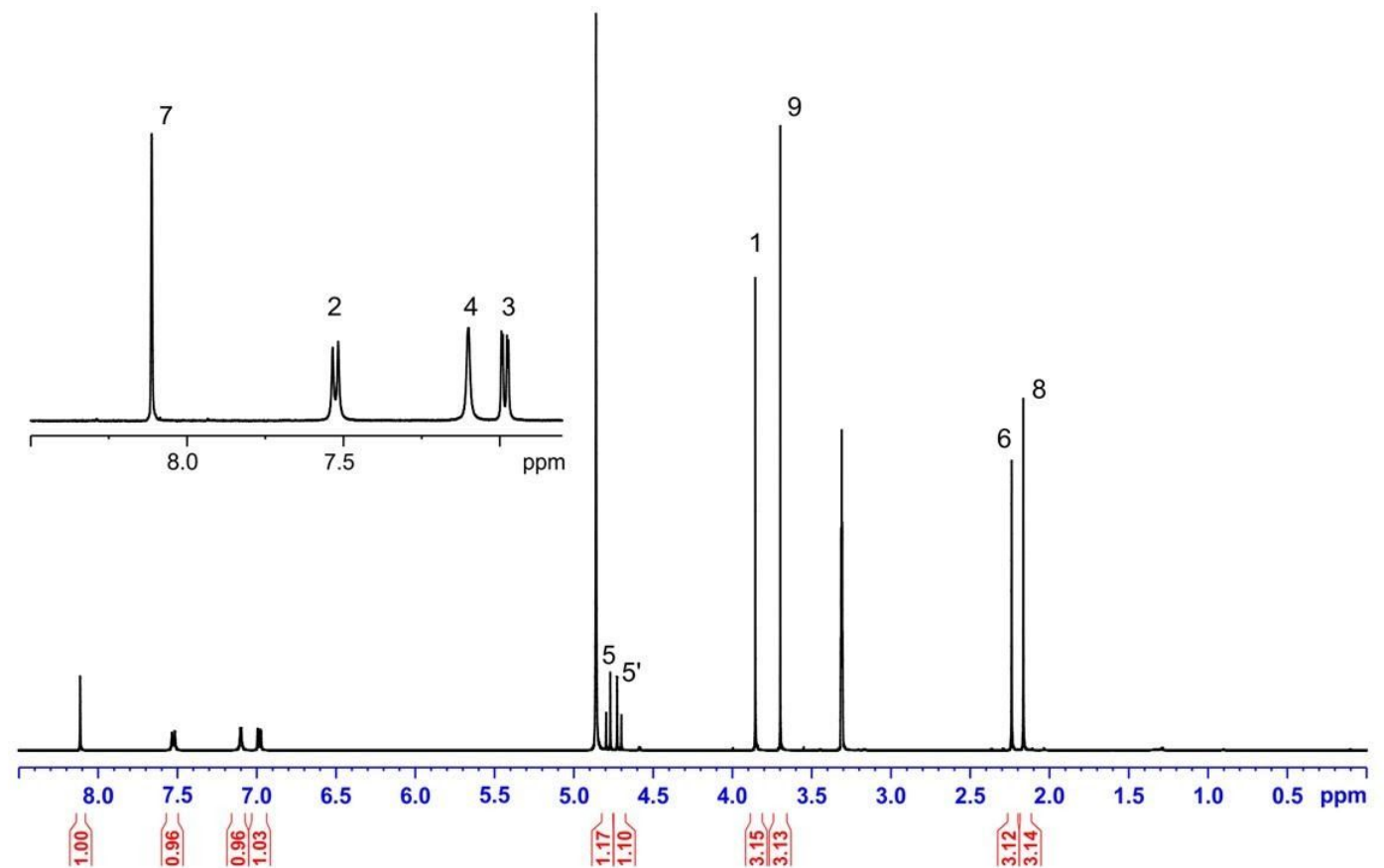

Fig. 5. ${ }^{1} \mathrm{H}$ NMR of omeprazole pure standard used to represent in details the peaks assignment (500 MHz, MeOD, 298K).

Proton nuclear magnetic resonance of the three internet omeprazoles analysed was compared to a European pharmaceutical reference sample is reported in Fig. 6. The NMR spectra for the samples analyzed was similar but with a few elements of differentiation. Characteristic peaks at 8.2, 7.5, 7.1 and 7.0 ppm were observed corresponding to omeprazole protons 7, 2, 4 and 3 respectively, as reported by Claramunt et al in an assessment study conducted using omeprazole standard [24]. These peaks are present in all the different classes of samples analyzed. Samples $\mathrm{C}$ and D showed two singlets at 7.6 and $7.7 \mathrm{ppm}$, respectively, close to the omeprazole doublet at $7.5 \mathrm{ppm}$ assigned to proton 2. These two singlets are indicative of additional aromatic molecules present as impurities. The region between 6.5 and $4.75 \mathrm{ppm}$ is where the strongly de-shielded methyl/methylene protons directly attached on the pyridinylic moieties could be found. The signals located in the area around $5 \mathrm{ppm}$ are related to the hydroxyl groups present in the excipients (as identified from comparison against reference samples shown in supplementary materials Figure S3), mainly lactose, cellulose and starch for all the formulations studied. 
The ${ }^{1} \mathrm{H}$ NMR analyses of the generic classes of omeprazole investigated present some remarkable differentiation (Fig. 6). Samples E and B showed the same formulation, with slightly different ratios (see SI), while samples $\mathrm{C}$ and $\mathrm{D}$ resulted to be identical in every aspect.

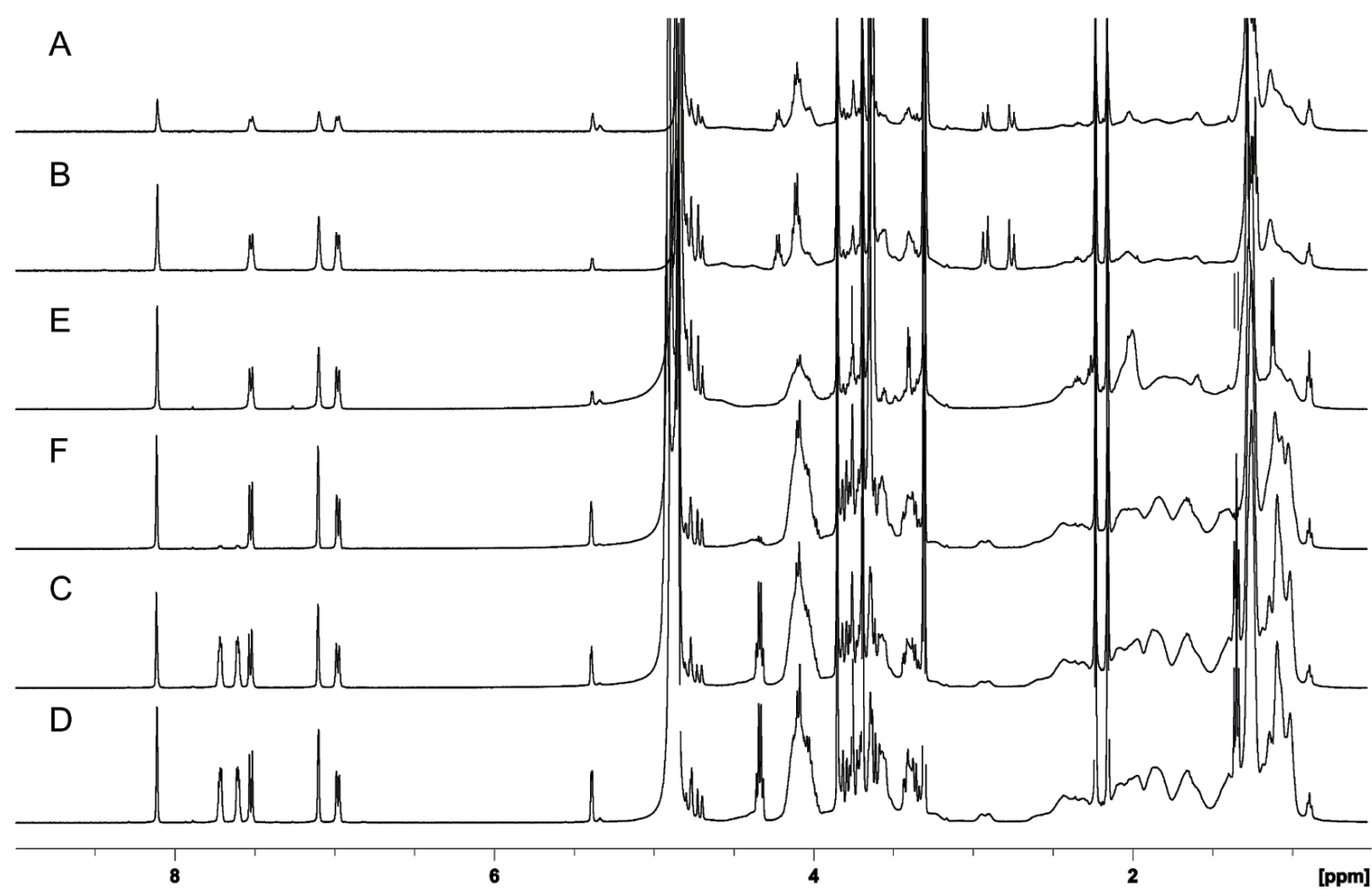

Fig. 6. ${ }^{1} \mathrm{H}$ NMR of the omeprazoles (500 MHz, MeOD, 298K). From top to bottom samples: A, B, E, F, C and D. At 7.6 and 7.7 ppm, samples C and D present two singlets associated with aromatic compounds different than omeprazole structure.

In general, all samples analyzed resulted to be very similar with similar amounts of both lactose and cellulose. However, unexpectedly, samples C and D contain extra signals in the aromatic region that most likely correspond to some impurity given that common excipients used on omeprazole formulations have no aromatic protons.

\subsubsection{Study of the internet samples $\mathrm{C}$ and $\mathrm{D}$.}

The ${ }^{1} \mathrm{H}$ NMR spectra obtained from samples $\mathrm{C}$ and D showed two signals at 7.6 and 7.7 ppm not found in the other samples that can be related to additional aromatic substances not present in the other formulations. It could be speculated that these aromatic signals 
correspond to impurities produced during the synthetic process of the omeprazole active pharmaceutical ingredient. Another possible cause for the presence of these impurities is that they originated as contamination from the specific manufacturing process for samples $\mathrm{C}$ and D. There are several omeprazole known impurities that are produced during the synthetic process and therefore methodology to identify its presence in omeprazole samples has been developed [25]. A comparison of the structure of known omeprazole impurities with the ${ }^{1} \mathrm{H}$ NMR of samples $\mathrm{C}$ and D hinted at 4-Desmethoxy omeprazole (also known as omeprazole impurity B) as being the compound responsible for the extra two singlets in the aromatic region of samples $\mathrm{C}$ and D. Indeed a reverse-phase LC-MS analysis of samples C and D (Figures S4 and S5 in supplementary material) identified the presence of a compound with $\mathrm{m} / \mathrm{z}=316$ Da that matches with the mass of 4-Desmethoxy omeprazole (see SI). Again the LC-MS traces were identical for samples $\mathrm{C}$ and $\mathrm{D}$, indicating that both samples have an extremely similar formulation.

Notwithstanding the possible causes that have produced the two aromatic impurities, their presence in the composition of the final pharmaceutical products, ready to be used by patients, raises suspects on the quality of the medications themselves. These two sets of samples have been both purchased off internet market from un-official websites, not regulated by any government authorization for the dispensing of medications. Samples $\mathrm{C}$ and $\mathrm{D}$ were purchased from the Asian region, through a European available website. In this geographic area, between 1999 and 2004, the ratio of fraudulent medications boosted drastically, with an increased of forged medications sales range from $10 \%$ to more than $30 \%$ of the national legal market. Because of the anonymous nature of the online market, the risks of entry into the market of medications of uncertain quality is remarkably high, being the quality control performed by the government authorities significantly more difficult and consequently less effective.

The manufacturing production of medicines is extremely articulated but it can be divided into two principal stages: the primary manufacturing stage, where the active pharmaceutical ingredient is manufactured, and the secondary manufacturing stage, where the active pharmaceutical ingredient (API) is mixed with other ingredients to prepare the final pharmaceutic formulation. Impurities in samples D and C could have derived from one or both of these stages. 
During the very last World Health Assembly, it was agreed to embrace the definition of 'Substandard and Falsified medical products' as an official term to be used by the member states with a most recent change in terminology introducing the classification of 'substandard' and 'falsified'. The first defines those official medical products that lack reaching the required standards of quality and/or to meet the necessary specifications. Falsified identifies those medical products that intentionally give false information on their nature, origin and structure. It is not clear at this stage of research if $\mathrm{D}$ and $\mathrm{C}$ could be classified as substandard or, worse, as falsified, but it seems probable that the lack of quality control that has allowed to input into the market these medicinals, containing aromatic products, and as such usually biochemically active, suggests addressing these products with caution.

\section{Discussion}

The analysis of the seven different PPIs does not give significantly distinctive spectra when studied via conventional Raman spectroscopy, both internally and externally. Using SERS, however, it was possible to observe an improvement in the characterisation of the spectra, especially on the analyses of the surfaces of the specimens. Although an omeprazole standard was not used in this study, previously published spectra compared to those obtained in this investigation showed that there are differences that can be accredited to the excipients added to the formulations [26-28]. All the spectroscopic analyses obtained from the internal and external parts of the samples were quite alike although some differences in quality and resolution could be noticed, due to a possible coating effect on the tablets. Previous spectroscopic studies reported that the concentration of active ingredients in solid formulations resulted to be non-homogeneously spread across from external to internal areas [29, 30]. SERS analyses of the internal side seem to show the same peaks but more pronounced than the external area. This could be related to the manufacturing processing, effectively giving a higher concentration at the internal area compared to that of the surfaces.

The spectra obtained from all the spectroscopic investigations did not produce a neat visual differentiation amongst the seven classes of samples investigated. It could be speculative to suggest that solid formulations often contain very low doses of API in 
comparison with the dosage of excipients used, which can present analytical challenges in terms of differentiation [30]. From the patient information leaflets, the list of excipients were similar with the only difference of hypromellose for the preparations $\mathrm{F}$ and G. Active pharmaceutical ingredients (APIs) can exist in different polymorph states, each one with specific chemical and physical properties which can affect directly their clinical pharmacological features. Manufacturer processing, handling, and storage can affect the polymorphic form state of the solid medication. Transformations can additionally occur due to interactions of APIs with excipients. Pharmaceutical solid forms can incorporate surface coating agents, with the function to act on the drug release performances or to improve the swallowability, and signals arising from such coating agents can cover API's ones. The thickness of the coating has a direct effect on the swamping of the API's signal too [31]. Numerous APIs include aromatic functional groups in their structure, which produce symmetric vibrational modes making the molecule strong Raman scatterers. On the contrary, many excipients are aliphatic, with modest Raman activity, which can masks the APIs Raman response [32]. The weights of the tablets for all the different formulations were ranging between 399 and $410 \mathrm{mg}$, leading to a ratio API/excipients of only 0.05 . The limited differentiation in the results obtained from the spectroscopic analyses was taken into consideration as a possible result obtained, because of the similar nature of the samples investigated. This was the justification to use chemometric analysis.

Cellulose, lactose and starch are the main excipients used in all of the formulations studied and all present a high ratio of hydroxyl groups, which could be masking with their broad peaks components of the omeprazole molecules. This is substantiated by the asymmetric stretching C-O-C and C-OH peaks found in the region around $1100 \mathrm{~cm}^{-1}$ from the sugar rings of the excipients [33, 34]. Notwithstanding the great resemblances amongst the spectra of the seven different classes of analytes, the statistical analysis of these spectroscopic data allowed the minor differences to be significative, even though only very few differences could be effectively being exploited. There were limited regions of the spectra obtained that could be associated with the individual omeprazole core structure. This could be because both the core of the molecules for all the classes of omeprazole is the same and that the excipients employed in the manufacturing of the 
solid formulations, covering any of the more noticeable differences, are indeed the same or belonging to sugar classes with same functional groups.

The scoreplots generated with the different Principal Components gave a visual clustering of the different PPIs, but, indeed, only little separations could be observed. Throughout PCA analysis of all spectroscopic investigations, the PPIs did not separate and clustered tightly, but they were rather spread in the same areas, overlapping. Only in the PCA obtained from the SERS analysis of the external areas, B, F and G showed to be clustered in separated groups. This evidence is in line with previous articles that reported how the enhanced sensitivity of SERS over conventional Raman leads to an improved selectivity which would allow for better discriminatory abilities $[35,36]$. The PCA variability ranged around $98-99 \%$ for all the categories of set of experiments performed.

In this case, cross-validation and leave-one-out used to validate the accuracy of the predictive models gave non-positive outcomes, with a considerable amount of error. The total error rates for all the PCAs executed were well over $75 \%$, both from cross-validation and from leave-one-out evaluation, showing that the multivariate analysis in this study did not present good discrimination power. There was no strong spectroscopic evidence in the factor loadings for the omeprazole formulations (not included). This is not unexpected given spectral interferences from the excess bulk agents present in the formulation already mentioned. These results substantiate the fact that the different PPI formulations studied are extremely similar.

Because of the need for additional information on the chemical structures of these samples to be able to discriminate differences amongst them, the use of NMR was considered to elucidate more chemical diversities. While Raman spectroscopy directly targets each part of the molecular structure of a given compound providing information related to the functional groups present, NMR gives information on the chemical structure of the analyte. NMR analyses of the seven different PPI preparations investigated presented some remarkable point of discrimination. E and B showed same formulation, with slightly different ratios. C, D, E, and B resulted to be very similar, with a similar amount of both lactose and cellulose. On the contrary, sample A seemed to be the only one not containing cellulose. F and G, both Levo-isomers of omeprazole racemate, contained the same chemicals, however, G contained considerably less API in 
the formulation. Both presented the presence of lactose and cellulose. G and F contain another aliphatic compound, probabily methacrylate, not found in the other classes of samples. Samples C and D resulted to be identical in each other, however, when compared to the rest of the samples contain an extra aromatic compound. The two singlets identified in the aromatic region of their ${ }^{1} \mathrm{H}$ NMR spectra were assigned to 4-Desmethoxy omeprazole using LC-MS and were otherwise not found in the other formulations. Samples C and D were purchased from uncertified online websites, and the parcels received were both coming from Asian regions. Because of the nature of the impurities tracked in these two generic medications, it is plausible to consider them as suspicious and potentially fraudulent products.

\section{Conclusions}

In this study, it was shown that Raman with the aid of chemometric methods did not offer enough analytical resolution to differentiate the different PPIs studied.

Further investigation by proton NMR of the backbone of the molecular structure of the PPI formulations highlighted the similarity of the samples, with $\mathrm{C}$ and $\mathrm{D}$ being in effect the same product, probably manufactured by the same plant and then commercialised under two different marketing names. E and B resulted nearly identical, showing a very similar NMR diagram, as well.

NMR investigation allowed to notice in the internet samples $C$ and D the presence of aromatic impurities, not detected during the Raman analysis. The problem of the uncertain quality of medications purchased online has been previously emphasised in this study, showing how probable it could be to obtain low quality medications when bought from un-authorised online sources.

These results are particularly relevant highlighting the fact that spectroscopic techniques, such as Raman, typically used in standard quality control tests in the pharmaceutical industry may miss impurities in medications produced in different manufacturer plants under different quality regimes. This opens the possibility to adopt 
NMR in the list of analytical technique that can help to assure the quality of medicines, tackling the problem of falsified and substandard medications.

\section{Acknowledgements}

This research received no specific grant from any funding agency in the public, commercial, or not-for-profit sectors

\section{References}

[1] B. Wallmark, Mechanism of Action of Omeprazole, J Scand Gastroent, 1986(21), pp $11-16$

[2] D. McTavish, M. Buckley, R.Heel, Omeprazole. An updated review of its pharmacology and therapeutic use in acid-related disorders, Drugs, 1991(42), pp $138-70$

[3] P. Miner, P.O. Katz, Y. Chen, M. Sostek, Gastric acid control with esomeprazole, lansoprazole, omeprazole, pantoprazole, and rabeprazole: a five-way crossover study, Am J Gastroenterol. 2003(12), pp 2616-20

[4] P. Meredith, Bioequivalence and other unresolved issues in generic drug substitution, Clin Ther 2003(25), pp 2875-2890

[5] N.D. Yeomans, Z. Tulassay Z, L. Juhász, I. Rácz, J.M. Howard, C.J. van Rensburg, A.J. Swannell, C.J. Hawkey, A comparison of omeprazole with ranitidine for ulcers 
associated with nonsteroidal antiinflammatory drugs, Acid Suppression Trial:

Ranitidine versus Omeprazole for NSAID-associated Ulcer Treatment (ASTRONAUT) Study Group. N Engl J Med. 1998(338), pp 719-26.

[6] P. Ekström, L. Carling, S. Wetterhus, P. E. Wingren, O. Anker-Hansen, G. Lundegårdh, E. Thorhallsson, P. Unge, Prevention of Peptic Ulcer and Dyspeptic Symptoms with Omeprazole in Patients Receiving Continuous Non-Steroidal Anti-Inflammatory Drug Therapy: A Nordic Multicentre Study, J Scand Gastroent, 1996, pp 753-758

[7] J.F.Riemann, D.Schilling, P. Schauwecker, G. Wehlen, D. Dorlars, B. Kohler, M. Maier, Cure with omeprazole plus amoxicillin versus long-term ranitidine therapy in Helicobacter pylori-associated peptic ulcer bleeding, Gastrointest Endoscop, 1997(46-4), pp 299-304

[8] T. Shimatani, M. Inoue, T. Kuroiwa, J. Xu, H. Mieno, S. Tazuma, Acid-suppressive effects of generic omeprazole: Comparison of three brands of generic omeprazole with original omeprazole, Digestive Liver Dis, 2006(38), Issue 8, pp 554-559

[9] T. Shimatani, S. Hirokawa, Y. Tawara, K. Hamai, M. Matsumoto, S. Tazuma, M. Inoue, Comparing the Acid-Suppressive Effects of Three Brands of Generic Lansoprazole with the Original: Pharmacokinetic Bioequivalence Tests Do Not Necessarily Guarantee Pharmacodynamic Equivalence, Dig Dis Sci, 2009(54), pp $2385-2390$

[10] J.E. Richter, S.M. Sabesin, D.G. Kogut, R.M. Kerr, L.D. Wruble, M.J. Collen, Omeprazole Versus Ranitidine or Ranitidine/Metoclopramide in Poorly Responsive Symptomatic Gastroesophageal Reflux Disease, Amer J Gastroent 1996(91-9), pp $1766-1772$ 
[11] B. Kaplan-Machlis, G. E. Spiegler, M. W. Zodet, D. A. Revicki, Effectiveness and costs of omeprazole vs ranitidine for treatment of symptomatic gastroesophageal reflux disease in primary care clinics in West Virginia, Archives of family medicine, 2000(9-7), pp 624

[12] P. N. Maton, R. Orlando, B. Joelsson, Efficacy of omeprazole versus ranitidine for symptomatic treatment of poorly responsive acid reflux disease - a prospective, controlled trial, Aliment Pharmacol Ther, 1999(13), pp 819-826

[13] www.abpi.org.uk/what-we-do/working-with-government-and-parliament/ falsified-medicines-directive-fmd/(accessed 01 April 2019)

[14] A. Surendran, World agencies try to stem flood of fake drugs, Nature Med, 2004(10), pp 111

[15] M. M. Cheng, Is the Drugstore Safe? Counterfeit Diabetes Products on the Shelves J Diab Sci Tech, 2009(3-6), pp 1516-1520

[16] M.C. Moken, Fake pharmaceuticals: how they and relevant legislation or lack thereof contribute to consistently high and increasing drug prices, Am J Law Med, 2003 (29), pp 525-542

[17] World Health Organization. 2014. Medicines:spurious/falsely-labelled/falsified/ counterfeit(SFFC)medicines.http://www.who.int/mediacentre/factsheets/fs275/en/. Accessed 16 January 2014.

[18] A. Attaran , D. Barry, S. Basheer, R. Bate, D. Benton, J. Chauvin, L. Garrett, I. Kickbusch, J.C. Kohle, K. Midha, P.N. Newton, S. Nishtar, P. Orhii, M. McKee, How to achieve international action on falsified and substandard medicines. BMJ345:e7381. 10.1136/ bmj.e7381.2012 
[19] A. Locniskar, D. J. Greenblatt, J. S. Harmatz, R. I Shader, Bioinequivalence of a generic brand of diazepam, Biopharm Drug Dispos, 1989(10), pp 597-605

[20] A. Guberman, Céline Corman, Generic Substitution for Brand Name Antiepileptic Drugs: A Survey, Can J Neurol Sci, 2000(27), pp 37-43

[21] T. Moore, A. Smith, W. Ye, D. Y. Toler, B. J. Westenberger, R. Lionberger, A. Raw, L. Yu, and L. F. Buhse, Generic omeprazole delayed-release capsules: in vitro performance evaluations, Drug Dev Ind Pharm, 2009(35), pp 917-921

[22] E. Smith, G. Dent, Modern Raman Spectroscopy, A practical approach, Wiley, 2005

[23] M. de Veij, P. Vandenabeele, T. De Beer, J.P. Remonc, L. Moensa, Reference database of Raman spectra of pharmaceutical excipients J. Raman Spectrosc, 2009(40) pp 297-307

[24] R. M. Claramunt, C. Lopez, I. Alkorta, J. Elguero, R. Yang, and S. Schulman, The tautomerism of omeprazole in solution: a ${ }^{1} \mathrm{H}$ and ${ }^{13} \mathrm{C}$ NMR study, Magn Reson Chem 2004(42), pp 712-714

[25] S. Koppala, V.R. Reddy, J.S. Anireddy, Development and Validation of a Novel Stability-Indicating RP-HPLC Method for the Simultaneous Determination of Related Substances of Ketoprofen and Omeprazole in Combined Capsule Dosage Form, J Chromatogr Sci, 2016(54-5), pp 765-75.

[26] D. A. Jackson, Stopping Rules in Principal Components Analysis: A Comparison of Heuristical and Statistical Approaches, Ecology, 1993(74), pp 2204-2214

[27] K. Buckley, P. Matousek, Recent advances in the application of transmission Raman spectroscopy to pharmaceutical analysis, J Pharm Biomed Anal, 2011(55), pp $645-652$ 
[28] P. V. Huong, Drug analysis by Raman and micro-Raman spectroscopy, J Pharm Biomed Anal, 1986(4), pp 811-823

[29] S. Han, and P. Faulkner, Determination of SB 216469-S during tablet production using near-infrared reflectance spectroscopy, J Pharm Biomed Anal, 1996(14-12), pp $1681-1689$

[30] Y. Roggo, P. Chalus, L. Maurer, C. Lema-Martinez, A. Edmond, N. Jent, A review of near-infrared spectroscopy and chemometrics in pharmaceutical technologies, $\mathrm{J}$ Pharm Biomed Anal, 2007(44), pp 683-700.

[31] Zhang GG, Law D, Schmitt EA, Qiu Y. Phase transformation considerations during process development and manufacture of solid oral dosage forms. Advanced drug delivery reviews 2004; 56: 371-390.

[32] C.J. Strachan, T. Rades T, K.C. Gordon, J. Rantanen, Raman Spectroscopy for Quantitative Analysis of Pharmaceutical Solids, J Pharm Pharmacol, 2007(59), pp 179-192

[33] D. Mayo et al., Course notes on the interpretation of infrared and Raman spectra, Wiley-Interscience, 2003

[34] S. Pînzaru, I. Pavel, N. Leopold, W. Kiefer, Identification and characterisation of pharmaceuticals using Raman and surface-enhanced Raman scattering. J Raman Spectr, 2004(35-5), pp 338-346

[35] P. Mosier-Boss, S. Lieberman, Detection of Nitrate and Sulfate Anions by Normal Raman Spectroscopy and SERS of Cationic-Coated, Silver Substrates. Appl Spectrosc, 2000(54-8), pp 1126-1135 
[36] K. Sun, Q. Huang, G. Meng, Y. Lu, Highly Sensitive and Selective Surface-Enhanced Raman Spectroscopy Label-free Detection of 3,3',4,4'-Tetrachlorobiphenyl Using DNA Aptamer-Modified Ag-Nanorod Arrays. ACS Appl Mater Interfaces, 2016(8-8), pp 5723-5728. 